\title{
Modélisation par séries de Fourier du fonctionnement en régime permanent d'un ensemble machine-convertisseur
}

\author{
H. Kateb et F. Rioux-Damidau \\ Laboratoire d'Electrotechnique des Universités Paris VI et XI, Unité associée au CNRS n 845, 91405 Orsay, \\ France
}

(Reçu le 2 juin 1987, révisé le 21 octobre 1987, accepté le 30 octobre 1987)

\begin{abstract}
Résumé. - Cet article décrit la modélisation à l'aide de séries de Fourier temporelles du fonctionnement en régime permanent d'un ensemble machine-convertisseur, pour une machine ayant un comportement linéaire par rapport au courant. Les résultats sont appliqués à une machine asynchrone sans fer.
\end{abstract}

Abstract. - This article describes the modelling by means of time Fourier series of the steady state operation of a motor-invertor system, for a motor having a linear behaviour with the current. The results are applied to an iron free asynchronous machine.

\section{Introduction.}

Aujourd'hui, les machines sont habituellement alimentées par des convertisseurs. Leur optimisation ne peut donc être réalisée de façon correcte que si l'on considère l'ensemble complet machine-convertisseur, car ce dernier représente en général une partie importante du système, et même une partie prépondérante lorsque le facteur de puissance de la machine est faible. Pour modéliser cet ensemble, il faut donc a priori résoudre simultanément les équations du champ dans la machine et celles du circuit du convertisseur $[1,2,3]$.

Cependant, si l'on s'intéresse seulement au fonctionnement en régime permanent et si l'on a affaire à une machine ayant un comportement linéaire en courant, les deux problèmes peuvent être résolus indépendamment. La représentation de la machine peut alors se faire simplement à l'aide d'impédances associées à chaque harmonique du courant. Dans certains cas, comme celui des machines asynchrones sans fer, ces impédances sont complètement calculables à partir des données géométriques, de la pulsation d'alimentation $\omega$ et de la vitesse de rotation $\Omega$ [4-7]. Il reste alors à résoudre les équations décrivant l'ensemble machine-convertisseur compte tenu des diverses impédances de la machine. C'est ce que nous avons fait en utilisant une décomposition en séries de Fourier temporelles des tensions et courants dans le système.

\section{Représentation de la machine.}

On considère une machine dont le stator est formé par un bobinage polyphasé classique. La première bobine de ce bobinage est alimentée par un courant $i_{1}(t)$ périodique, de pulsation $\omega$ qui peut être décomposé en séries de Fourier :

$$
i_{1}(t)=\sum_{n \in \mathbb{Z}} I_{n} \mathrm{e}^{i n \omega t} .
$$

La tension aux bornes de cette même bobine est :

$$
V_{1}(t)=\sum_{n \in \mathbb{Z}} V_{1 n} \mathrm{e}^{i n \omega t} .
$$

Les courants et tensions existant dans les autres bobines sont obtenus par simple déphasage des valeurs dans la première bobine.

Si la machine présente un comportement linéaire vis-à-vis du courant, c'est-à-dire si elle ne comporte pas de matériau magnétique saturé, on peut montrer que $V_{1 n}$ et $I_{n}$ sont proportionnels.

On écrit :

$$
V_{1 n}=\Lambda_{n} n \omega I_{n}
$$


$\Lambda_{n}$ est en quelque sorte une impédance complexe qui vérifie la relation :

$$
\Lambda_{-n}=-\Lambda_{n}^{*} \text {. }
$$

Dans le cas des machines asynchrones sans fer, les $\Lambda_{n}$ ont été calculés par une modélisation tridimensionnelle à base de séries de Fourier [7]. Nous avons montré qu'ils obéissent à des relations très simples qui permettent de les connaître tous à partir des quelques premiers. $\Lambda_{1}$ est particulier, il dépend essentiellement de la pulsation des courants rotoriques (soit $\omega-p \Omega$ pour une machine $2 p$-polaire dont la vitesse angulaire de rotation est $\Omega$ ) ; sa partie réelle est proportionnelle au couple et sa partie imaginaire à la puissance réactive. Les coefficients $\Lambda_{n}$ correspondant à $n$ pair sont nuls ; les autres (pour $n$ impair) sont indépendants de $\omega$ et très faciles à obtenir dès que l'on connaît les premiers. Dans le cas d'une machine bipolaire, il suffit de calculer (outre $\Lambda_{1}$ ) les trois coefficients $\Lambda_{3}, \Lambda_{5}$ et $\Lambda_{11}$ pour une vitesse de rotation $\Omega_{0}$ donnée et l'on a ensuite, pour la vitesse $\Omega$ :

$$
\begin{array}{r}
\left\{\begin{array}{l}
\operatorname{Re}\left(\Lambda_{n}\right)=\frac{\operatorname{Re}\left(\Lambda_{i^{\prime}}\right) \sqrt{i^{\prime} \Omega_{0}}}{\sqrt{n \Omega}} \\
\operatorname{Im}\left(\Lambda_{n}\right)=\operatorname{Im}\left(\Lambda_{i^{\prime}}\right)
\end{array}\right. \\
\text { avec }\left\{\begin{array}{l}
n=12 k \pm i, k \in \mathbb{N}, i=1,3,5 \\
i^{\prime}=i \text { pour } i=3 \text { ou } 5 \\
i^{\prime}=11 \text { pour } i=1 .
\end{array}\right.
\end{array}
$$

\section{Le convertisseur.}

On considère ici un convertisseur alimentant une machine hexaphasée [8] constitué de deux pontsmachine (pont $A$ et pont $B$ ) triphasés et décalés de $30^{\circ}$ (cf. Fig. 1). Il fournit une onde de courant de pulsation $\omega$ formée de deux arches de forme pseudotrapézoïdale, l'une positive, l'autre négative, séparées par des intervalles de courant nul (Fig. 3a). L'inductance de lissage $L$ est suffisante pour considérer que le courant est constant entre deux commutations (égal à $\pm I$ ou nul). Durant la commutation entre deux phases, il dépend des paramètres de l'ensemble machine-convertisseur. Les deux pontsmachines $\mathrm{A}$ et $\mathrm{B}$ sont identiques à un déphasage de $\pi / 6 \omega$ près des courants et tensions. Il nous suffira donc d'étudier le fonctionnement du pont $\mathrm{A}$.

$\mathrm{Au}$ cours d'une période, on observe six commutations qui sont toutes identiques. Nous avons choisi de faire l'étude de la commutation du courant de la phase 1 à la phase 2 . Elle se compose des séquences classiques suivantes :

- Avant la commutation, le courant passe par le thyristor Th1, la diode D1, traverse la phase 1 et la phase 3, puis retourne à la source par la diode D2 et le thyristor $\mathrm{Th} 2$.

- Durant la séquence « $0 »$ s'effectue la commutation du courant du thyristor Th1 au thyristor Th3. La durée de cette séquence est tellement courte qu'elle sera négligée dans la suite du calcul.

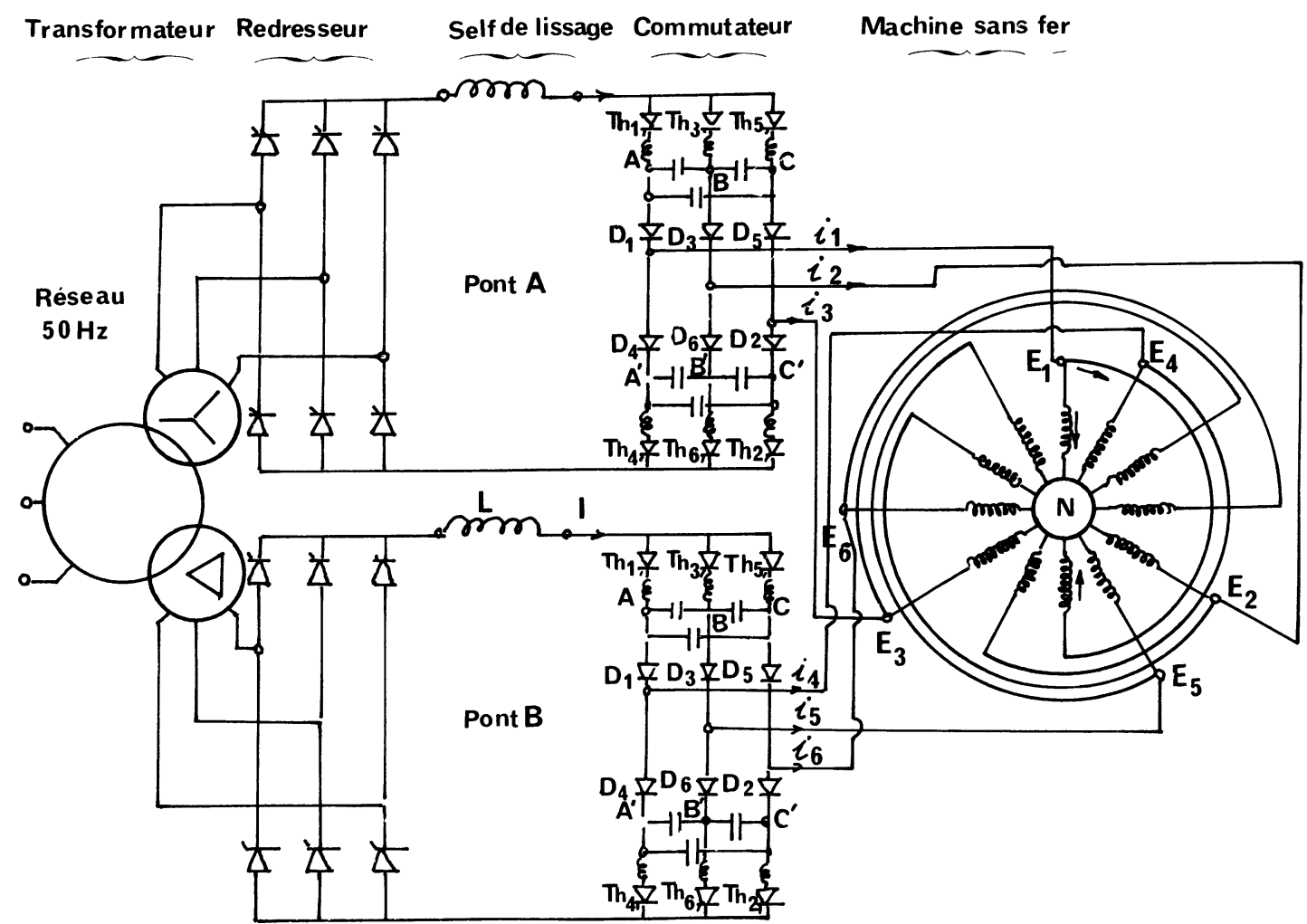

Fig. 1. - Ensemble machine-convertisseur.

[The motor-invertor system.] 
- Pendant la séquence « $I$ », qui commence à l'instant $t=-t_{1}$, les courants $i_{1}=I, i_{2}=0, i_{3}=$ - $I$ restent constants. Les courants dans les condensateurs sont aussi constants et valent :

$$
i_{\mathrm{C}_{1}}=-\frac{2}{3} I, \quad i_{\mathrm{C}_{3}}=i_{\mathrm{C}_{5}}=\frac{I}{3} \text {. }
$$

La tension aux bornes de $\mathrm{C}_{1}$ à la fin de cette séquence, c'est-à-dire à l'instant $t=0$ vaut ainsi :

$$
V_{\mathrm{BA}}(0)=V_{0}-\frac{2 I}{3 C} t_{1}
$$

$V_{0}$ étant la tension aux bornes de $C$ à l'instant $-t_{1}$.

- Enfin durant la séquence «II » figure 2, se produit la commutation effective du courant de la phase 1 à la phase 2. La tension aux bornes de $\mathrm{C}_{1}$ vaut :

$$
V_{\mathrm{BA}}(t)=V_{\mathrm{BA}}(0)+\frac{1}{C} \int_{0}^{t} i_{\mathrm{C}_{1}}(t) \mathrm{d} t
$$

avec $i_{\mathrm{C}_{1}}=-\frac{2}{3} i_{1}$ où $i_{1}$ n'est plus constant.

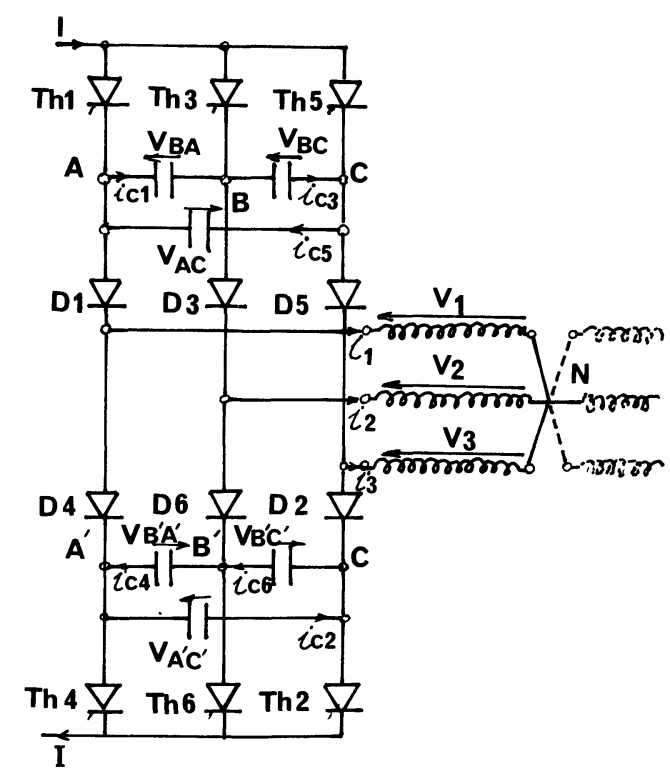

Fig. 2. - Passage du courant lors de la séquence de commutation effective du courant de la phase 1 à la phase 2 (séquence II).

[Current flow during the current's commutation sequence II.]

- Après la commutation, on obtient une séquence de fonctionnement de même type qu'avant la commutation, mais $i_{1}$ est remplacé par $i_{2}$.
Les condensateurs $C_{1}, C_{3}$ et $C_{5}$ à la fin de la commutation se trouvent chargés de manière à assurer la commutation qui suit, c'est-à-dire de la phase 2 à la phase 3.

\section{Modélisation du système.}

Nous admettons que le régime permanent est établi et que le déroulement des séquences est connu $a$ priori. Cela suppose que les conditions suivantes sont vérifiées $[10]$ :

- L'ordre d'enclenchement des thyristors suit l'ordre de leur numérotation.

- Lors du passage du courant d'un thyristor vers l'autre dans un même demi-convertisseur (exemple séquence « $0 »)$, le troisième reste bloqué.

- En dehors des commutations, deux thyristors seulement peuvent conduire simultanément.

- Un thyristor ne peut conduire plus de $2 \pi / 3 \omega$ durant une période $T=2 \pi / \omega$.

Ainsi, l'ordre de succession des séquences de fonctionnement en régime permanent est fixé; le courant en dehors des commutations est connu; les seules inconnues du problème restent la forme du courant durant les séquences de commutation ainsi que la durée de ces dernières. Nous allons calculer ces grandeurs : nous vérifierons ensuite que l'évolution des tensions aux bornes des condensateurs ainsi que celle des tensions aux bornes des diodes et des thyristors sont telles qu'elles permettent le déroulement des séquences prévu.

4.1 CALCUl DU COURANT DURANT LA COMMUTATION. - Dans la séquence « I » le courant $i_{1}$ est constant, alors qu'il varie durant la séquence «II », nous l'appellerons courant de commutation $i_{\mathrm{c}}$ et il obéit à l'équation différentielle suivante :

$$
i_{\mathrm{c}}=i_{1}=-\frac{3}{2} C \frac{\mathrm{d} V_{\mathrm{BA}}}{\mathrm{d} t}
$$

avec

$$
i_{\mathrm{c}}(0)=I \quad \text { et } \quad \frac{\mathrm{d} i_{\mathrm{c}}}{\mathrm{d} t}(0)=0
$$

Or la tension aux bornes de $\mathrm{C}_{1}$ est alors égale à la différence des tensions aux bornes des phases de la machine en commutation, on a :

$$
V_{\mathrm{BA}}(t)=V_{1}-V_{2}=V_{12}
$$

où $V_{2}(t)$ est identique à $V_{1}(t)$ à un déphasage de $2 \pi / 3 \omega$ près (système triphasé équilibré). Par suite, en utilisant l'expression (3) donnant $V_{1 n}$ :

$$
V_{12}=\sum_{n \in \mathbb{Z}} n \omega I_{n} \Lambda_{n}\left(1-\mathrm{e}^{-i n 2 \pi / 3}\right) \mathrm{e}^{i n \omega t}
$$


Il faut bien remarquer que dans (12) $I_{n}$ est le coefficient de Fourier du courant total $i_{1}$ et non du seul courant de commutation $i_{\mathrm{c}}$ intervenant dans (11) : or $i_{1}$ peut être considéré comme la somme de créneaux connus de durée $2 \pi / 3 \omega$ et de 4 arches de formes identiques issues de $i_{\mathrm{c}}$ (voir Fig. 3). Compte tenu de la forme du courant $i_{1}$ on voit facilement que [9] :

$$
I_{n}=\left[1-(-1)^{n}\right]\left[1+\mathrm{e}^{-i n \pi / 3}\right]\left(\frac{i I}{2 \pi n}+I_{\mathrm{c}_{n}}\right)
$$

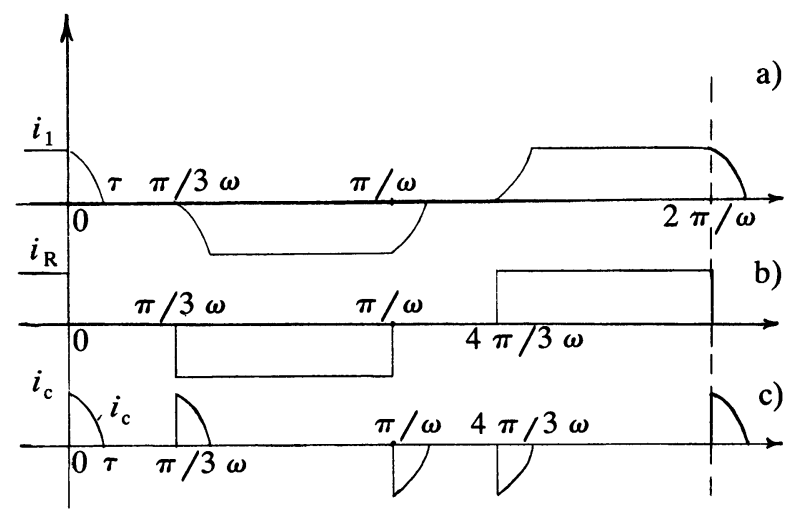

Fig. 3. - Le courant dans la $1^{\text {re }}$ phase $i_{1}(t)$ est la somme de créneaux $i_{\mathrm{R}}(t)$ et d'arches issues du courant de commutation $i_{\mathrm{c}}(t)$.

[The current flowing in phase 1 is the sum of levels $i_{\mathrm{R}}(t)$ and commutation arches $i_{\mathrm{c}}$.] où le coefficient de Fourier $I_{\mathrm{c}_{n}}$ de $i_{\mathrm{c}}$ est tel que :

$$
I_{\mathrm{c}_{n}}=-\frac{3 \omega C}{4 \pi} \int_{0}^{\tau} \frac{\mathrm{d} V_{12}}{\mathrm{~d} t} \mathrm{e}^{-i n \omega t} \mathrm{~d} t
$$

La relation (13) implique que pour $n$ multiple de 2 ou $3 I_{n}$ soit nul. L'ensemble des relations (12), (13) et (14) permet de déterminer $I_{n}$; cependant des difficultés apparaissent car $V_{12}$ présente une discontinuité au temps $\tau$ (Fig. 4).

4.2 SOLUTION APPORTÉE AUX PROBLÈMES POSÉS PAR LE SAUT DE TENSION. - Par suite du saut de $V_{12}$ à la fin de la commutation, la quantité $\frac{\mathrm{d} V_{\mathrm{BA}}}{\mathrm{d} t}=$ $\frac{\mathrm{d} V_{12}}{\mathrm{~d} t}$ est un pic de Dirac en $\tau$ qui est donc, en pratique, impossible à approximer par une série de Fourier. Pour éviter ce problème nous intégrons (14) par parties :

$$
\begin{aligned}
I_{\mathrm{c}_{n}}=-\frac{3 \omega C}{4} & {\left[V_{12}(\tau) \mathrm{e}^{-i n \omega \tau}-V_{12}(0)\right]-} \\
& -\frac{3 \omega C}{4 \pi} i n \omega \int_{0}^{\tau} V_{12}(t) \mathrm{e}^{-i n \omega t} \mathrm{~d} t .
\end{aligned}
$$

Dans cette expression, nous devrions remplacer $V_{12}$ par $V_{12}^{N}$, c'est-à-dire par la série de Fourier (12) limitée à l'ordre $N$. Cependant par suite du phéno-

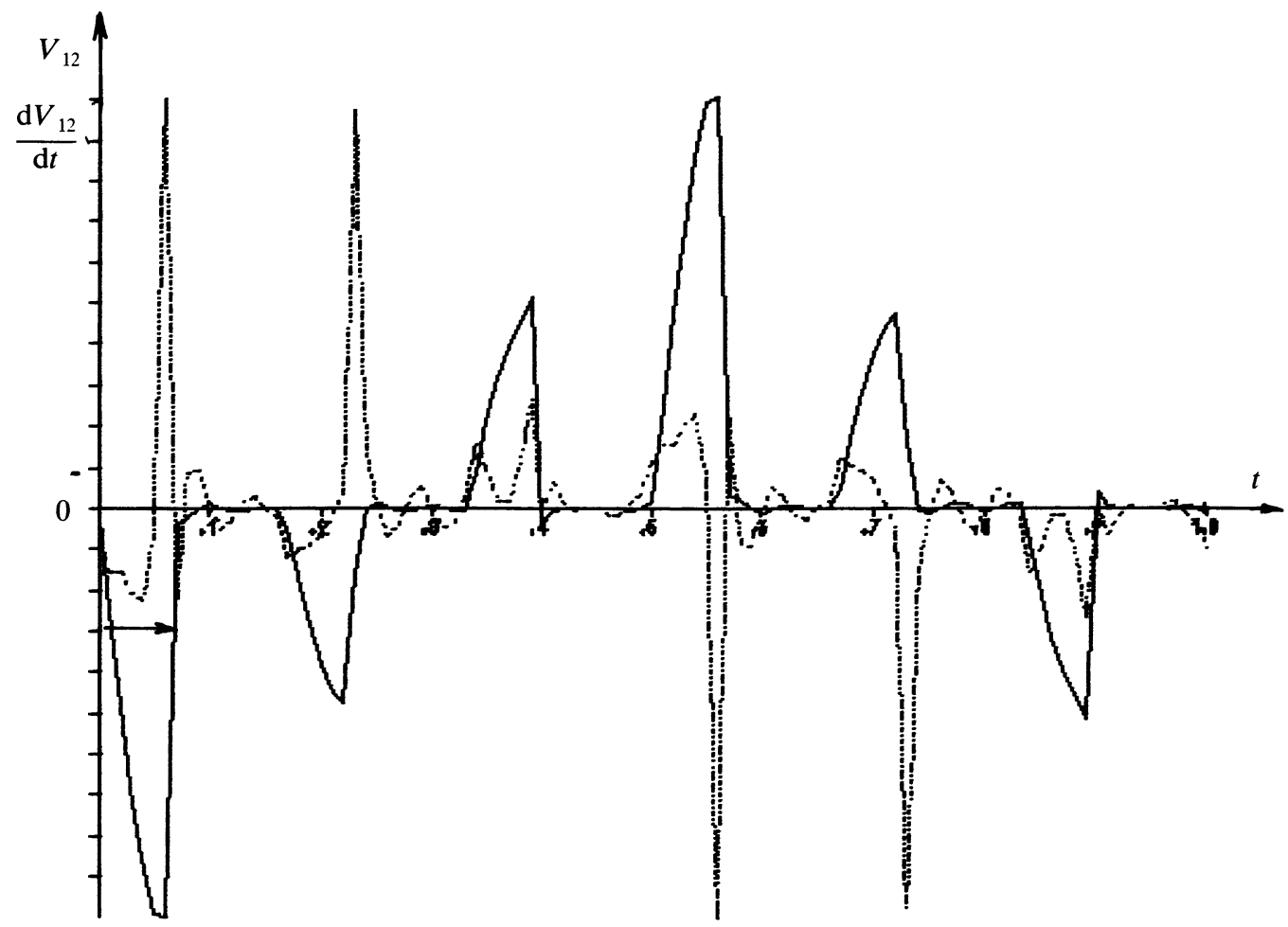

Fig. 4. - La tension $V_{12}=V_{1}-V_{2}$ (en trait plein) et sa dérivée $\mathrm{d} V_{12} / \mathrm{d} t$ en pointillés dans le cas d'une charge $(R, L)$.

[The voltage $V_{12}=V_{1}-V_{2}$ (solid line) and its derivative $\mathrm{d} V_{12} / \mathrm{d} t$ when loading $(R, L)$.] 
mène de Gibbs [9] (voir Fig. 5) ceci n'est pas correct à l'instant $\tau$ ni au voisinage de cet instant même si $N$ est grand. En effet, au point $\tau$ lui-même :

$$
V_{12}^{N}(\tau)=\left[V_{12}(\tau+\varepsilon)+V_{12}(\tau-\varepsilon)\right] / 2 .
$$

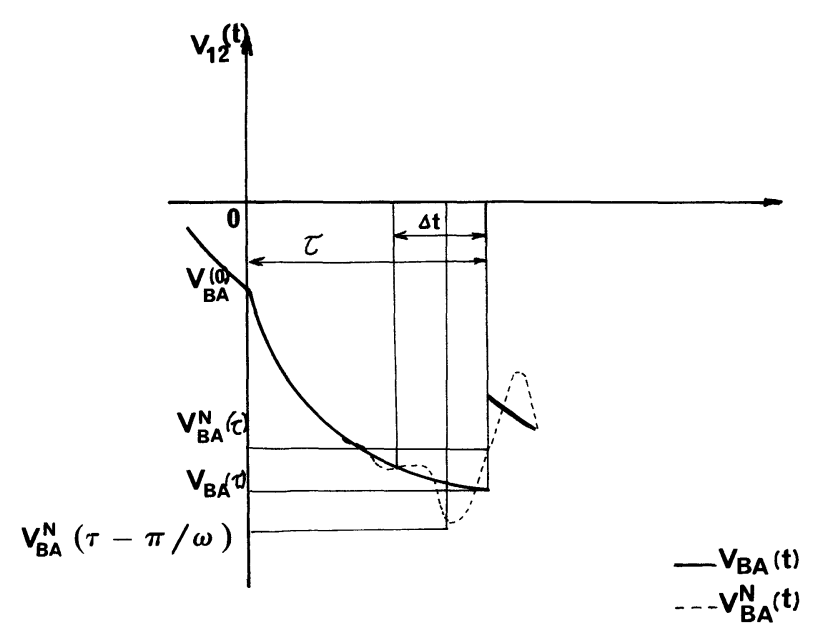

Fig. 5. - La représentation de $V_{\mathrm{BA}}=V_{1}-V_{2}$ par la série de Fourier limitée à l'ordre $N$ (trait plein) et sa valeur réelle (en pointillés) dans l'intervalle $(0, \tau)$.

[The representation of $V_{1}-V_{2}$ by its Fourier series limited to order $N$ (solid line) and its real value (dotted line).]

De plus, au voisinage de $\tau, V_{12}^{N}$ oscille. Les extréma de l'oscillation ont lieu pour $t=\tau \pm \pi / 2 N \omega$ et ils dépassent le saut lui-même d'environ $18 \%$. Aussi, dans (15), nous remplacerons $V_{12}(\tau)$ par l'expression suivante :

$V_{12}(\tau)=\frac{\left[V_{12}^{N}(\tau-\pi / N \omega)-V_{12}^{N}(\tau)\right]}{1,178}+V_{12}^{N}(\tau)$.

La forme oscillante de $V_{12}^{N}(t)$ au voisinage de $\tau$ affecte beaucoup la valeur de l'intégrale apparaissant dans (15).

$$
\int_{0}^{\tau}=\int_{0}^{\tau-\Delta t}+\int_{\tau-\Delta t}^{\tau}
$$

Jusqu'à l'instant $\tau-\Delta t, V_{12}^{N}$ oscille peu et diffère peu de $V_{12}$; il peut donc représenter une bonne approximation de $V_{12}$.

Entre $\tau-\Delta t$ et $\tau, V_{12}$ ne peut plus être représentée par $V_{12}^{N}$ qui oscille trop; nous avons préféré l'approximer par la fonction :

$$
V_{12}(\tau) \sin \omega_{0} t
$$

où $\omega_{0} \tau=\pi / 2$ et $V_{12}(\tau)$ est donné par (17).
4.3 RELATIONS SATISFAITES PAR LES COEFFICIENTS DE FOURIER $I_{n}$. - Calculant $I_{c_{n}}$ par (15) avec les approximations indiquées au paragraphe précédent et reportant son expression dans (13), on obtient les équations satisfaites par les $I_{n}$ ( $n$ n'étant multiple ni de 2 , ni de 3 ce qui implique qu'il soit tel que $n=6 k \pm 1, k \in \mathbb{Z})$.

$$
I_{n}+\sum_{n^{\prime}=-N}^{N} A_{n, n^{\prime}} I_{n^{\prime}}=B_{n}
$$

où :

$$
\begin{aligned}
& A_{n, n^{\prime}}= \frac{3 \omega^{2} C}{2}\left(1+\mathrm{e}^{-i n \pi / 3}\right)\left(1-\mathrm{e}^{-i n^{\prime} 2 \pi / 3}\right) \times \\
& \times n^{\prime} \Lambda_{n^{\prime}}\left\{\mathrm{e}^{i\left(n^{\prime}-n\right) \omega \tau}\left[\frac{\left(\mathrm{e}^{-i n^{\prime} \pi / N}-1\right)}{1,178}+1\right] \times\right. \\
& \times\left\{1-\frac{n \omega}{2}\left[\frac{1-\mathrm{e}^{i\left(\omega_{0}+n \omega\right) \Delta t}}{\omega_{0}+n \omega}-\right.\right. \\
&\left.\left.-\frac{1-\mathrm{e}^{-i\left(\omega_{0}-n \omega\right) \Delta t}}{\omega_{0}-n \omega}\right]\right\}- \\
&\left.-1+\frac{n}{n^{\prime}-n}\left[\mathrm{e}^{i\left(n^{\prime}-n\right)(\omega \tau-\omega \Delta t)}-1\right]\right\} \\
& B_{n}=\left(1+\mathrm{e}^{-i n \pi / 3}\right) \frac{i I}{\pi n} .
\end{aligned}
$$

Comme le courant $i_{1}(t)$ est réel :

$$
I_{n}=I_{-n}^{*} .
$$

Les deux inconnues complexes $I_{n}$ et $I_{-n}$ ne sont donc pas indépendantes et ne correspondent qu'à deux inconnues réelles. Posant :

$$
\begin{aligned}
& I_{n}=X_{n}^{\prime}+i X_{n}^{\prime \prime} \\
& B_{n}=Y_{n}^{\prime}+i Y_{n}^{\prime \prime} .
\end{aligned}
$$

On déduit de (23) un système d'équations réelles :

$$
\begin{aligned}
X_{n}^{\prime}+ & \sum_{n^{\prime}>0}^{N}\left\{\operatorname{Re}\left[A_{n, n^{\prime}}(\tau)+A_{n,-n^{\prime}}(\tau)\right] X_{n^{\prime}}^{\prime}+\right. \\
& \left.+\operatorname{Im}\left[A_{n,-n^{\prime}}(\tau)-A_{n, n^{\prime}}(\tau)\right] X_{n^{\prime}}^{\prime \prime}\right\}=Y_{n}^{\prime} \\
X_{n}^{\prime \prime}+ & \sum_{n^{\prime}>0} \operatorname{Im}\left[A_{n, n^{\prime}}(\tau)+A_{n,-n^{\prime}}(\tau)\right] X_{n^{\prime}}^{\prime}+ \\
& \left.+\operatorname{Re}\left[A_{n, n^{\prime}}(\tau)-A_{n,-n^{\prime}}(\tau)\right] X_{n^{\prime}}^{\prime \prime}\right\}=Y_{n}^{\prime \prime} .
\end{aligned}
$$

Posant $N=6 K-1($ ou $6 K+1)$, on voit que ce système comporte $4 K$ (ou $4 K+2$ ) équations à $4 K$ (ou $4 K+2$ ) inconnues réelles ; on l'écrira sous forme matricielle :

$$
M(\tau, \Delta t) X=Y
$$

4.4 VALIDITÉ DE LA MODÉLISATION TESTÉE POUR UNE ChARge $R L$. - Pour tester la validité de la modélisation que nous venons de décrire, nous l'avons appliquée au cas où le convertisseur alimente 
un système formé de six bobines simples ayant une inductance $L$ et une résistance $R$. Dans ce cas, on sait calculer la solution analytique qui sert alors de référence.

Le système (24) dépend du temps de commutation $\tau$ et du temps $\Delta t$ servant dans le calcul de l'intégrale (18). $\Delta t$ est en principe choisi « convenablement », $\tau$ est une inconnue du problème.

Dans le cas d'une charge $R L$, la valeur analytique de $\tau$ est connue. Nous l'avons introduite dans (24) et nous avons résolu ce système pour diverses valeurs de $\Delta t$ (couvrant au maximum quelques oscillations de $\left.V_{12}^{N}\right)$. Il apparaît qu'avec $\Delta t=\pi / N \omega$, nous obtenons une bonne valeur du courant (les calculs ont été faits pour trois valeurs différentes de $N: 59$,

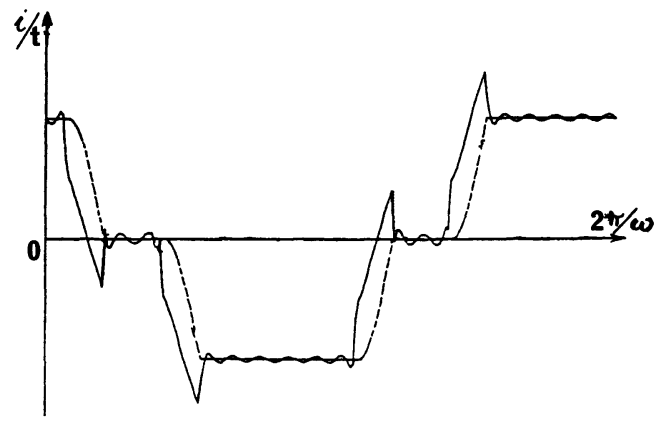

Fig. 6. - Modélisation du courant $i_{1}(t)$ dans le cas d'une charge $(R, L)$; avec $\Delta t=0$ (en trait plein) et $\Delta t=$ $\pi / N \omega$ (en pointillés), $N=179$.

[Modelling of the invertor current $i_{1}(t)$ when loading $(R, L)$ with $\Delta t=0$ (solid line) and $\Delta t=\pi / N \omega$ (dotted line), $N=179$.]
119 et 179). Nous adopterons donc désormais cette valeur de $\Delta t$. La figure 6 montre, pour $N=179$, le courant calculé avec $\Delta t=0$ et $\Delta t=\pi / \omega$; elle fait apparaître clairement l'intérêt de la décomposition (18) de l'intégrale.

On constate cependant que, malgré la superposition des courbes donnant le courant réel et le courant calculé par la méthode de Fourier, les coefficients de Fourier correspondant sont très proches pour $n \ll N$ mais diffèrent de plus en plus au fur et à mesure que $n$ augmente (cf. Tab. I). Cela n'est pas grave puisque ces coefficients deviennent de plus en plus petits lorsque $n$ s'accroît. Cette difficulté provient du fait que les harmoniques $n>N$ ont été négligés dans le système (24).

La valeur de $\Delta t$ étant fixée, nous avons cherché à déterminer par une méthode itérative la valeur de $\tau$ qui satisfait simultanément les conditions aux limites suivantes :

$$
\text { et } \quad \begin{aligned}
\sum_{n=-N}^{+N} I_{n} & =I \\
\sum_{n=-N}^{+N} I_{n} \mathrm{e}^{i n \omega \tau} & =0 .
\end{aligned}
$$

Nous avons ainsi retrouvé la valeur analytique de $\tau$ permettant d'obtenir une forme de courant convenable.

\section{Etude de l'ensemble machine-convertisseur.}

5.1 DÉTERMINATION DES CARACTÉRISTIQUES. La modélisation de la machine donne les $\Lambda_{n}$. Lorsqu'on les reporte dans (24), on peut résoudre ce

\begin{tabular}{|c|c|c|}
\hline $\begin{array}{c}\text { Numéro } \\
\text { d'harmonique } n\end{array}$ & Méthode analytique & $\begin{array}{l}\text { Méthode par les } \\
\text { séries de Fourier }\end{array}$ \\
\hline 1 & $(7,6708574 ; 7,8531266)$ & $(7,7070436 \quad ; \quad 7,8239148)$ \\
\hline 5 & $(1,3206095 ; 1,4634602)$ & ; 1,4534880$)$ \\
\hline 7 & $(0,94324888 ;-0,83587826)$ & $(0,94240018 ;-0,87109359)$ \\
\hline 11 & $(0,40963089 ;-0,38800026)$ & $(0,41960219 ;-0,40743496)$ \\
\hline 13 & $(-0,27305528 ;-0,25230911)$ & $(-0,28365841 \quad ;-0,26292786)$ \\
\hline 17 & $(-0,14117190 ;-0,054009736)$ & $(-0,13880884 \quad ;-0,05847931)$ \\
\hline$\vdots$ & & \\
\hline 73 & $(0,00482632 ;-0,00698001)$ & $(0,0063571117 ;-0,00912065)$ \\
\hline 77 & $(0,00117423 ;-0,00773092)$ & $(0,003063640 ;-0,00791701)$ \\
\hline 79 & $(-0,00720415 ; \quad 0,000614082)$ & $(-0,0064474039 ;-0,00619765)$ \\
\hline$\vdots$ & & \\
\hline 173 & $(0,00020952 ;-0,0014913757)$ & $(-0,0031672539 ;-0,00258997)$ \\
\hline 175 & $(-0,00144511 ; 0,0001595360)$ & $(-0,0024534301 ; \quad 0,002348435)$ \\
\hline 179 & $(-0,00107725$ & $(0,004669330 ;-0,001697472)$ \\
\hline
\end{tabular}

Tableau I. - Coefficients de Fourier du courant réel et du courant calculé à partir de (24).

[Fourier coefficients of the real current and of the current calculated from (24).] 
système par itération en prenant $\Delta t=\pi / N \omega$ et obtenir ainsi les $I_{n}$. On peut alors calculer un certain nombre de caractéristiques du système [9].

La tension de précharge $V_{0}$ s'écrit immédiatement :

$$
\begin{aligned}
V_{0}=-V_{12}(\tau) & =-\sum_{n \in \mathbb{Z}} n \omega \Lambda_{n} I_{n}\left(1-\mathrm{e}^{-i n 2 \pi / 3}\right) \times \\
& \times \mathrm{e}^{i n \omega \tau}\left[\frac{\left(\mathrm{e}^{-i n \pi / N}-1\right)}{1,178}+1\right] \cdot(27)
\end{aligned}
$$

La durée $t_{1}$ de la séquence «I» de commutation vérifie la relation (10) soit encore :

$V_{12}(0)=\sum_{n \in \mathbb{Z}} n \omega \Lambda_{n} I_{n}\left(1-\mathrm{e}^{i n 2 \pi / 3}\right)=V_{0}-\frac{2 I}{3 C} t_{1}$.

Les expressions (27) et (28) permettent de déduire :

$$
\begin{aligned}
t_{1}=-\frac{3 C}{2 I} & \sum_{n \in \mathbb{Z}} n \omega \Lambda_{n} I_{n}\left(1-\mathrm{e}^{-i n 2 \pi / 3}\right) \times \\
\times & \left\{1+\mathrm{e}^{i n \omega \tau}\left[\frac{\left(\mathrm{e}^{-i n \pi / N}-1\right)}{1,178}+1\right]\right\}
\end{aligned}
$$

On peut aussi calculer le temps de repos des thyristors ; c'est le temps $t_{i}$ durant lequel, après blocage, ils subissent une tension négative à leurs bornes. Lors de la séquence « 0 » à l'amorçage de Th3, c'est-à-dire à partir du temps $t \simeq-t_{1}$, le thyristor Th1 est soumis à la tension $-V_{\mathrm{BA}}(t)$ donnée par (6) ; celle-ci négative au départ, s'annule au temps $t_{i}$ tel que :

$$
t_{i}=\frac{3 C V_{0}}{2 I}
$$

Cette valeur doit être 20 à $50 \%$ plus élevée que le temps de désamorçage $t_{\mathrm{q}}$ du thyristor [10].

Le rôle principal des condensateurs $\mathrm{C}_{1}$ à $\mathrm{C}_{6}$ est de fournir l'énergie nécessaire au moment opportun pour forcer la commutation du courant d'une phase vers l'autre de la machine. L'évolution des tensions à leurs bornes est liée à l'état des circuits régissant chaque séquence de fonctionnement de l'ensemble machine-convertisseur.

La tension aux bornes de $\mathrm{C}_{1}$ est définie par les fonctions suivantes [10] :

$$
V_{\mathrm{BA}}(t)= \begin{cases}V_{0}-\frac{2 I}{3 C} t_{1}-\frac{2}{3 C} \int_{0}^{\tau} i_{1}(t) \mathrm{d} t & ; 0 \leqslant \omega t \leqslant \omega \tau \\ -V_{0} & ; \omega \tau \leqslant \omega t \leqslant \frac{2 \pi}{3}-\omega t_{1} \\ -V_{0}+\frac{I}{3 C}\left[t-\left(\frac{2 \pi}{3 \omega}-t_{1}\right)\right] & ; \frac{2 \pi}{3}-\omega t_{1} \leqslant \omega t \leqslant \frac{2 \pi}{3} \\ -V_{0}+\frac{I}{3 C} t_{1}+\frac{1}{3 C} \int_{\frac{2 \pi}{3 \omega}}^{t} i_{2}(t) \mathrm{d} t & ; \frac{2 \pi}{3} \leqslant \omega t \leqslant \frac{2 \pi}{3}+\omega \tau \\ 0 & ; \frac{2 \pi}{3}+\omega \tau \leqslant \omega t \leqslant \frac{4 \pi}{3}-\omega t_{1} \\ I\left[t-\left(\frac{4 \pi}{3 \omega}-t_{1}\right)\right] & ; \frac{4 \pi}{3}-\omega t_{1} \leqslant \omega t \leqslant \frac{4 \pi}{3} \\ \frac{I t_{1}}{3 C}+\frac{1}{3 C} \int_{\frac{4 \pi}{3 \omega}}^{t} i_{3}(t) \mathrm{d} t & ; \frac{4 \pi}{3} \leqslant \omega t \leqslant \frac{4 \pi}{3}+\omega \tau \\ +V_{0} & ; \frac{4 \pi}{3}+\omega \tau \leqslant \omega t \leqslant 2 \pi-\omega t_{1} \\ V_{0}-\frac{2 I}{3 C}\left[t-\left(\frac{2 \pi}{\omega}-t_{1}\right)\right] & ; 2 \pi-\omega t_{1} \leqslant \omega t \leqslant 2 \pi .\end{cases}
$$

Sachant que,

$$
\begin{aligned}
& i_{2}(t)=\sum_{n \in \mathbb{Z}} I_{n} \mathrm{e}^{i n(\omega t-2 \pi / 3)} \\
& i_{3}(t)=\sum_{n \in \mathbb{Z}} I_{n} \mathrm{e}^{i n(\omega t-4 \pi / 3)}
\end{aligned}
$$

nous calculons le coefficient de Fourier complexe $V_{\mathrm{BA}_{n}}$ qui permet ensuite de recomposer $V_{\mathrm{BA}}(t)$ :

$$
V_{\mathrm{BA}_{n}}=\frac{3 i \mathrm{e}^{-i n \omega \tau}}{2 \pi n}\left(V_{0}-\frac{I t_{1}}{3 C}\right)-\frac{I}{2 \pi C \omega n^{2}}\left(1-\mathrm{e}^{i n \omega t_{1}}\right)+\frac{1}{2 \pi C \omega} \sum_{n^{\prime} \in \mathbb{Z}} I_{n^{\prime}}\left(\frac{\mathrm{e}^{i\left(n^{\prime}-n\right) \omega \tau}-1}{n^{\prime}\left(n^{\prime}-n\right)}+\frac{\mathrm{e}^{-i n \omega \tau}-1}{n n^{\prime}}\right)
$$

avec $V_{\mathrm{BA}_{n}}=0$ pour $n=3 k$ où $k \in \mathbb{Z}$. 
La tension aux bornes de la diode d'isolement D1 est définie par les équations suivantes :

$$
V_{\mathrm{D} 1}(t)=\left\{\begin{array}{lll}
0 & \text { pour } & 0 \leqslant t \leqslant \tau \\
V_{2}-V_{1}+V_{\mathrm{BA}} & \text { pour } & \tau \leqslant t \leqslant \tau+\frac{2 \pi}{3 \omega} \\
V_{3}-V_{1}-V_{\mathrm{AC}} & \text { pour } & \tau+\frac{2 \pi}{3 \omega} \leqslant t \leqslant \frac{4 \pi}{3 \omega} \\
0 & \text { pour } & \frac{4 \pi}{3 \omega} \leqslant t \leqslant \frac{2 \pi}{\omega} .
\end{array}\right.
$$

On peut calculer les coefficients de Fourier correspondants. On trouve :

$$
\begin{aligned}
V_{\mathrm{D} 1 n}= & \frac{i}{2 \pi} \sum_{n^{\prime} \in \mathbb{Z}} \frac{1}{n^{\prime}-n}\left[V_{1 n^{\prime}}\left(\mathrm{e}^{-i n^{\prime} 2 \pi / 3}-1\right)+V_{\mathrm{BA}_{n^{\prime}}}\right] \mathrm{e}^{i\left(n^{\prime}-n\right) \omega \tau} \times \\
& \times\left[1-\mathrm{e}^{i\left(n^{\prime}-n\right) 2 \pi / 3}\right]+\frac{i}{2 \pi} \sum_{n^{\prime} \in \mathbf{Z}} \frac{1}{n^{\prime}-n}\left[V_{1 n^{\prime}}\left(\mathrm{e}^{-i n^{\prime} 4 \pi / 3}-1\right)-V_{\mathrm{BA}_{n^{\prime}}} \mathrm{e}^{-i n^{\prime} 4 \pi / 3}\right] \\
& \times\left[\mathrm{e}^{i\left(n^{\prime}-n\right)(\omega \tau+2 \pi / 3)}-\mathrm{e}^{i\left(n^{\prime}-n\right) 4 \pi / 3}\right] .
\end{aligned}
$$

Notons que les coefficients de Fourier sont non nuls pour toute valeur de $n$ y compris $0 . V_{1 n^{\prime}}$ et $V_{\mathrm{BA}_{n^{\prime}}}$ sont données par les formules (3) et (31) respectivement.

La tension aux bornes du thyristor Th1 est définie par :

$$
\begin{aligned}
& V_{\mathrm{T} 1}(t)= \\
& = \begin{cases}-V_{\mathrm{BA}} & \text { pour } 0 \leqslant t \leqslant 2 \pi / 3 \omega-t_{1} \\
V_{\mathrm{AC}} & \text { pour } 2 \pi / 3 \omega-t_{1} \leqslant t \leqslant 4 \pi / 3 \omega-t_{1} \\
0 & \text { pour } 4 \pi / 3 \omega-t_{1} \leqslant t \leqslant 2 \pi / \omega-t_{1} \\
-V_{\mathrm{BA}} & \text { pour } 2 \pi / \omega-t_{1} \leqslant t \leqslant 2 \pi / \omega .\end{cases}
\end{aligned}
$$

On en déduit le coefficient de Fourier :

$$
\begin{aligned}
V_{\mathrm{T} 1 n} & =-\frac{i}{2 \pi} \sum_{n^{\prime} \in \mathrm{Z}} \frac{V_{\mathrm{BA}_{n^{\prime}}} \mathrm{e}^{-i n \omega t_{1}}}{n^{\prime}-n} \times \\
& \times\left(\mathrm{e}^{i\left(n^{\prime}-n\right) 2 \pi / 3}-1\right)\left(\mathrm{e}^{-i\left(n+n^{\prime}\right) 2 \pi / 3}-1\right) .
\end{aligned}
$$

\subsection{EXISTENCE DU FONCTIONNEMENT DE L'ONDU-} LEUR. - L'ordre du déroulement des séquences de fonctionnement en régime permanent étant fixé $a$ priori, les conditions ci-dessous doivent être vérifiées pour qu'il ait effectivement lieu.

- Les diodes D3 et D5 doivent être bloquées juste avant la commutation du courant du thyristor Th1 au thyristor Th3, ce qui se traduit par:

$$
\begin{aligned}
& V_{\mathrm{D} 3}=V_{1}-V_{2}-V_{\mathrm{BA}}<0 \\
& V_{\mathrm{D} 5}=V_{1}-V_{3}+V_{\mathrm{AC}}<0 .
\end{aligned}
$$

Or à cet instant, $V_{\mathrm{BA}}=V_{0}$ et $V_{\mathrm{AC}}=-V_{0}$. Les inégalités ci-dessus conduisent à [10] :

$$
\sum_{n \notin \mathbb{Z}} n \omega \Lambda_{n} I_{n} \mathrm{e}^{-i n \omega t_{1}}<2 V_{0} / 3
$$

$$
\sum_{n \in \mathbb{Z}} n \omega \Lambda_{n} I_{n}\left(1-\mathrm{e}^{-i n 2 \pi / 3}\right) \mathrm{e}^{-i n \omega t_{1}}<V_{0} .
$$

- Au début de la commutation effective du courant de la phase 1 à la phase 2, la diode D3 doit entrer en conduction et pas la diode D5. On doit donc avoir $V_{\mathrm{D} 3}=0$ et $V_{\mathrm{D} 5}<0$. Reprenant les expressions (34) de $V_{\mathrm{D} 3}$ et $V_{\mathrm{D} 5}$ et sachant que les tensions $V_{\mathrm{BA}}(t)$ et $V_{\mathrm{AC}}(t)$ à la fin de la séquence «I » sont telles que :

$$
\begin{aligned}
& V_{\mathrm{BA}}(t)=V_{0}-\frac{2 I}{3 C} t \\
& V_{\mathrm{AC}}(t)=-V_{0}+\frac{I t}{3 C}
\end{aligned}
$$

on en déduit la condition :

$$
V_{2}(0)+V_{1}(0)-2 V_{3}(0)<V_{0}
$$

soit encore

$$
\sum_{n \in \mathbb{Z}}-3 n \omega \Lambda_{n} I_{n} \mathrm{e}^{-i n 4 \pi / 3}<V_{0} .
$$

Il faut finalement que les conditions (35), (36) et (37) soient satisfaites, donc en particulier la plus restrictive d'entre elles.

D'autre part l'alimentation de la machine étant constituée par deux ponts machines décalés l'un par rapport à l'autre de $30^{\circ}$, la durée de la dernière séquence de commutation doit satisfaire l'inégalité :

$$
\tau \leqslant \pi / 6 \omega .
$$

Sinon, il apparaît un empiètement de phases entre les deux ponts machines.

Enfin, pour que le cycle des diodes ne soit pas perturbé, il faut que

$$
t_{1}+\tau \leqslant 2 \pi / 3 \omega .
$$


5.3 EXEMPLE D'APPLICATION. - Il existe au Laboratoire d'Electrotechnique un prototype de moteur asynchrone sans fer délivrant $150 \mathrm{~kW}$ à $20000 \mathrm{tr} / \mathrm{min}$, dont la modélisation a été effectuée il y a déjà un certain temps et pour lequel les $\Lambda_{n}$ sont bien connus. Avec ces $\Lambda_{n}$, nous avons résolu (24) et obtenu le courant, puis tracé les $i_{1}(t)$ et $V_{21}(t)$ représentées sur la figure 7 . Les expres-

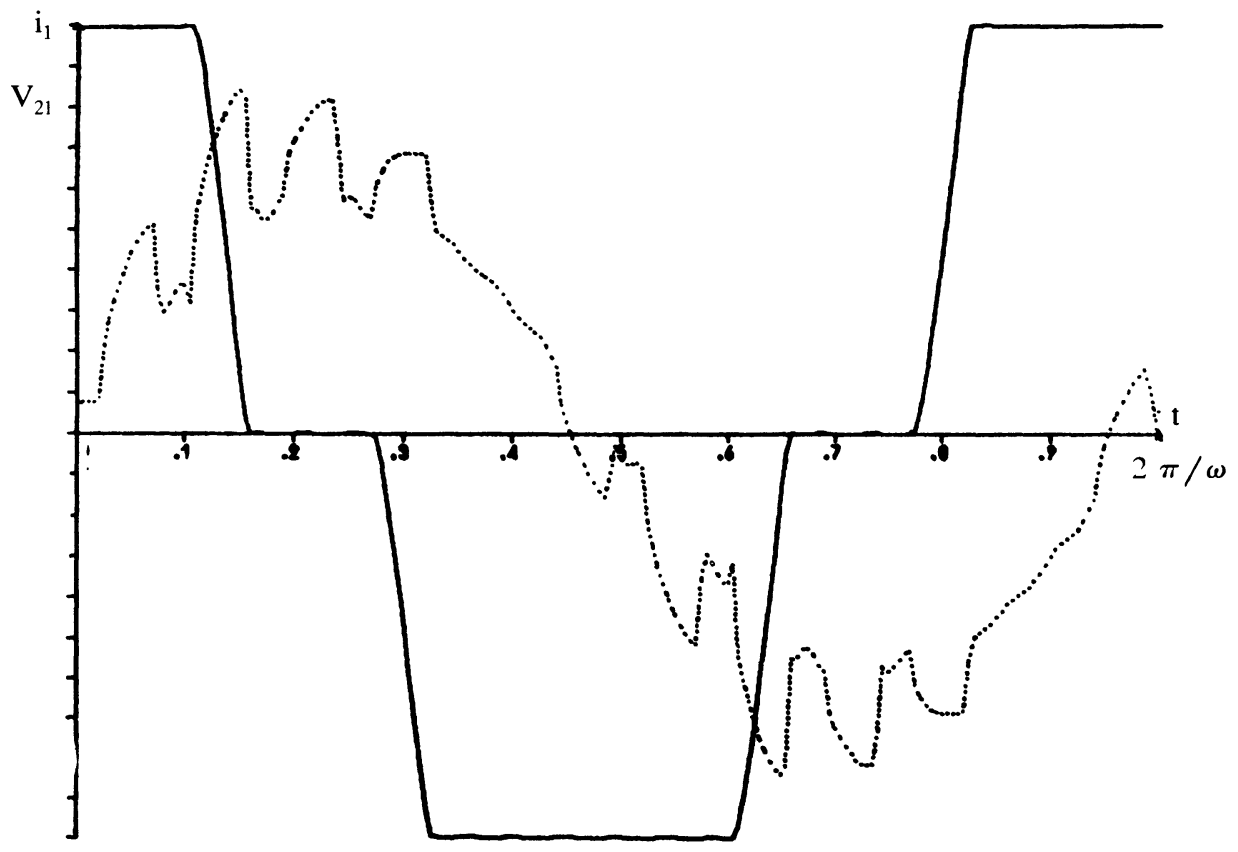

Fig. 7. - Modélisation du courant $i_{1}(t)$ (en trait plein) et de la tension $V_{21}(t)$ (en pointillés) dans le cas d'une machine asynchrone (prototype d'essai).

[Modelling of the current $i_{1}(t)$ (solid line) and the voltage $V_{2}-V_{1}$ at the asynchronous machine's hands.]

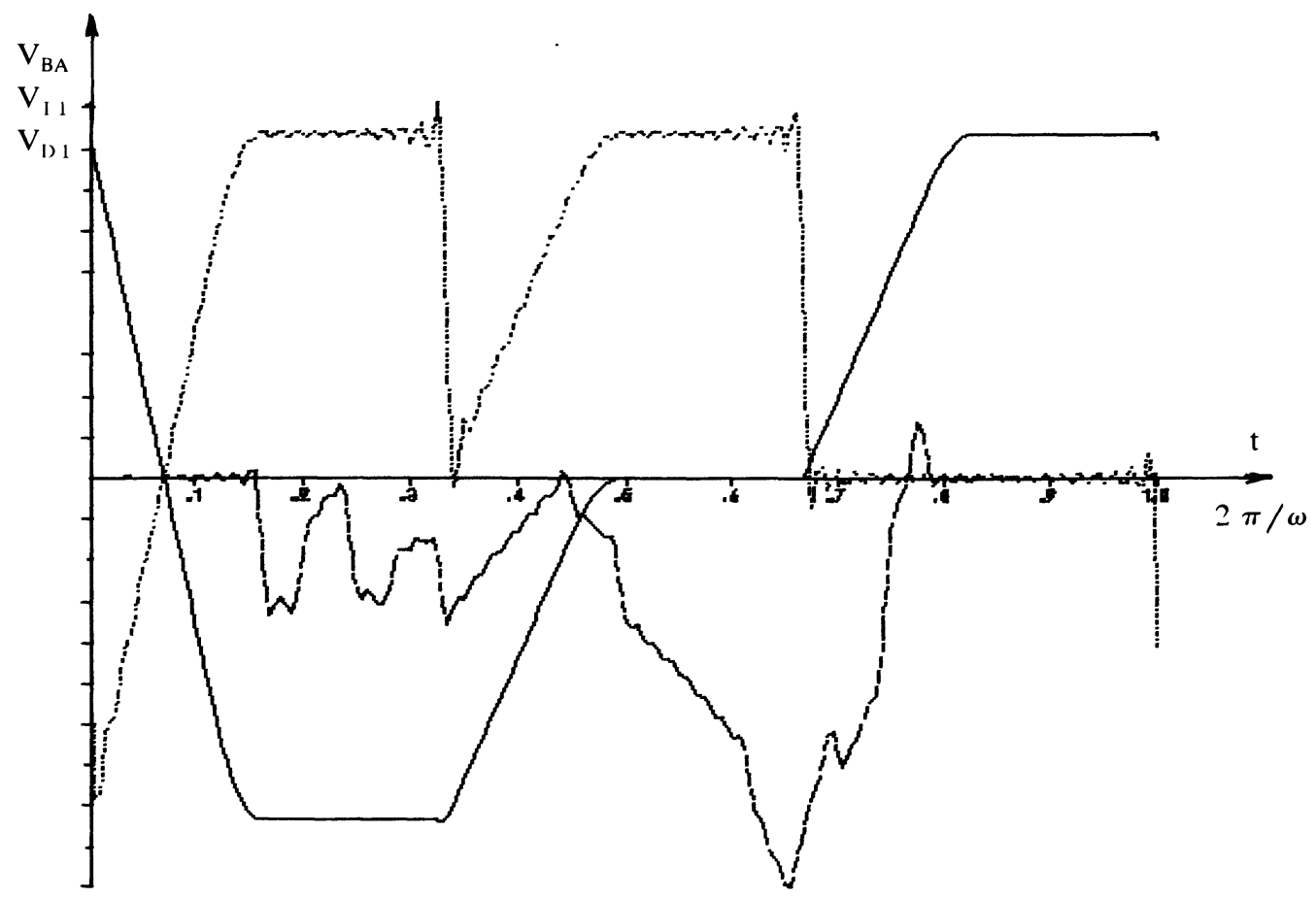

Fig. 8. - Modélisation de la tension aux bornes du condensateur $\mathrm{C} 1, V_{\mathrm{BA}}(t)$ (en trait plein), du thyristor Th1, $V_{\text {Th1 }}$ (en pointillés) et de la diode $V_{\mathrm{D} 1}$ (en tirets).

[Modelling of the voltage at the $\mathrm{C} 1$ capacitor hands (solid line), at the diode $\mathrm{D}_{1}$ hand (dotted line) and at the Th1 thyristor hands.] 
sions (31), (32) et (33) nous ont ensuite permis de calculer les tensions aux bornes des condensateurs, des diodes et des thyristors. Ces tensions sont représentées sur la figure 8 .

Nous avons ensuite étudié les caractéristiques $V_{0} / I$ (rappelons que le système est linéaire en $I), t_{1}, \tau$ et $t_{i}$ en fonction de la fréquence d'alimentation du stator $\nu$, de la pulsation rotorique $\nu_{\mathrm{R}}$ et de la valeur de la capacité $C$ utilisée dans le convertisseur.

La figure 9.1 montre ces grandeurs en fonction de $\nu$ pour $\nu_{\mathrm{R}}=4,5 \mathrm{~Hz}$ (valeur pour laquelle le couple est maximal) et $C=180 \mu \mathrm{F}$ (valeur de la capacité dans le convertisseur existant). On voit que le temps de commutation $\tau$ reste presque constant et que les autres grandeurs augmentent légèrement avec $\tau$.

La figure 9.2 donne l'évolution des mêmes grandeurs en fonction de $\nu_{R}$ pour une fréquence typique de fonctionnement, $350 \mathrm{~Hz}$, et toujours pour $C=$ $180 \mu \mathrm{F}$. On distingue deux plages de valeurs $\nu_{\mathrm{R}}$ : la première s'étend de 0 à $5 \mathrm{~Hz}$, elle correspond au fonctionnement habituel et les caractéristiques $V_{0} / I, t_{1}$ et $t_{i}$ diminuent rapidement avec l'augmentation de $\nu_{\mathrm{R}}$; dans la plage suivante (au-delà de $15 \mathrm{~Hz}$ ), elles se stabilisent et ne dépendent plus de $\nu_{\mathrm{R}}$. On constate aussi que le temps de commutation $\tau$ est pratiquement constant ; il passe par un maximum léger aux alentours de $\nu_{\mathrm{R}}=4,5 \mathrm{~Hz}$.

La figure 9.3 montre l'influence de la capacité $C$, pour $\nu_{\mathrm{R}}=4,5 \mathrm{~Hz}$ avec une alimentation du stator à $350 \mathrm{~Hz}$. La tension de précharge $V_{0}$ décroît lorsque $C$ augmente alors que $t_{1}, \tau$ et $t_{1}$ croissent.

\section{Conclusion.}

Une première étude de la modélisation par séries de Fourier d'un ensemble machine-convertisseur a été mise en place. Plusieurs problèmes ont dû être résolus. Ils sont essentiellement liés aux discontinuités des tensions qui apparaissent lors du fonctionnement des thyristors, discontinuités qui posent des difficultés lors de leur modélisation par les séries de Fourier (phénomène de Gibbs).

La méthode a tout d'abord été testée pour un ensemble convertisseur-charge $(R, L)$ pour lequel nous pouvions effectuer le calcul analytique du fonctionnement et disposer par suite d'une possibilité de vérification. Ayant ainsi obtenu une modélisation valable, nous l'avons appliquée à un ensemble machine sans fer-convertisseur, les machines sans fer ayant été modélisées par ailleurs à partir des équations des champs, en traitant celles-ci grâce à une décomposition en séries de Fourier spatio-temporelles des grandeurs physiques.
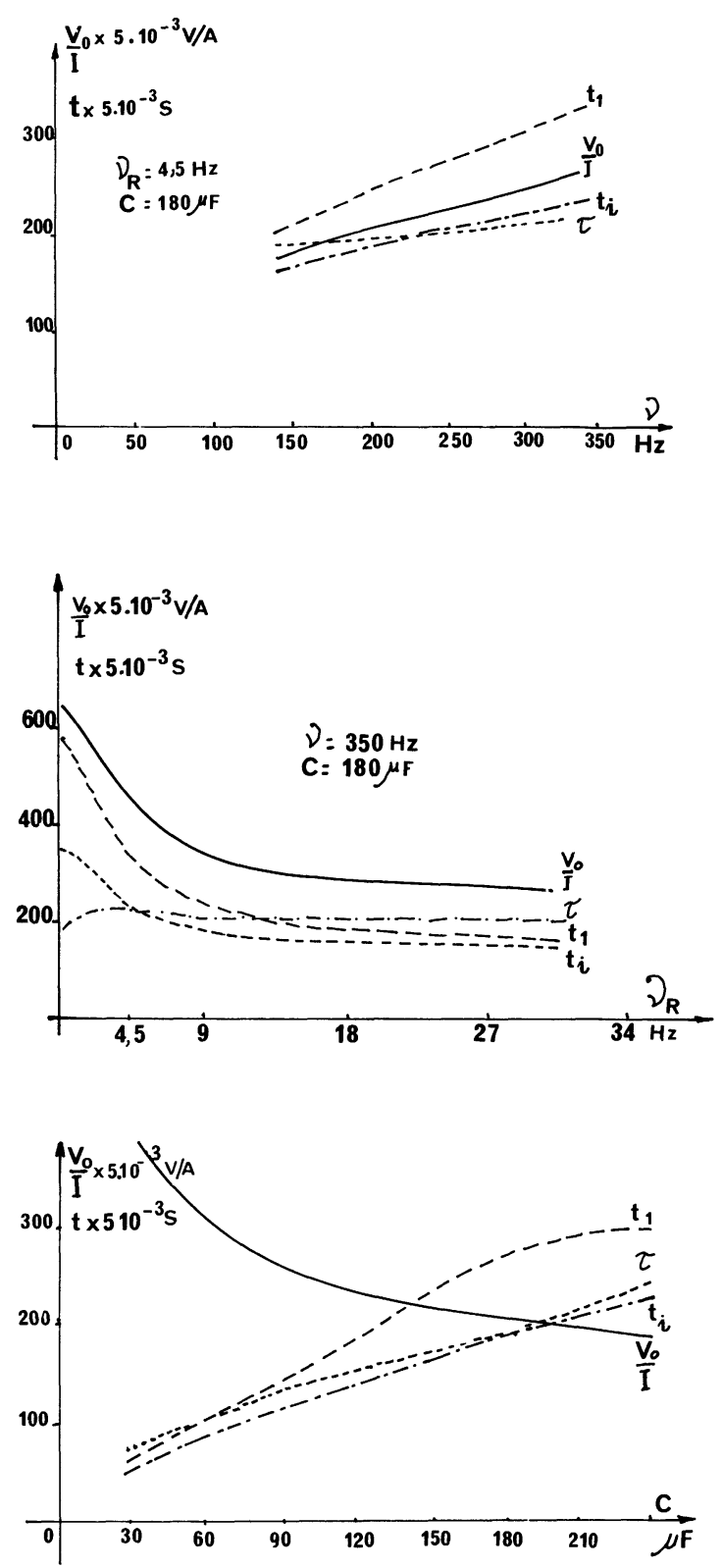

Fig. 9. - Variation de $V_{0} / I, \tau$ et $t_{i}$ en fonction de la fréquence d'alimentation $\nu$ (9.1), de la fréquence rotorique $\nu_{\mathrm{R}}(9.2)$ et de la capacité des condensateurs $C(9.3)$.

[Variation of $V_{0} / I, t_{1}, \tau$ and $t_{i}$ as a function of the alimentation frequency $\nu(9.1)$, of the rotor frequency $\nu_{\mathrm{R}}$ (9.2), of the capacity $C$ of an invertor branch.]

Ainsi, l'ensemble de la modélisation de la machine proprement dite et de son fonctionnement en régime permanent lorsqu'elle est alimentée par un convertisseur permet aujourd'hui l'optimisation du système machine asynchrone sans fer-convertisseur. 


\section{Bibliographie}

[1] Davat, B., Lajoie-Maz Enc, M. and Hector, J. Magnetic structure and circuit modeling, IEEE Trans. Magn. 19 (1983) 2471-2473.

[2] Journée d'étude organisée par le groupe S.E.E. Midi Pyrénées et GRECO-C.N.R.S. à L'ENSEEIHT de Toulouse 1985: Méthodes d'études et de modélisation des ensembles convertisseurs statiques-machines électriques.

[3] Desesquelles, P. F., Glaize, C., Simulation numérique d'ensembles machine à réluctance variable-convertisseur à thyristors, $R G E 4$ (1985) 282285.

[4] Rioux-Damidau, F., Calcul semi-tridimensionnel des champs et des courants dans les machines asynchrones sans fer. Revue Phys. Appl. 18 (1983) 113.

[5] Rioux-Damidau, F., Modélisation des machines cylindriques asynchrones sans fer. Prise en compte de la longueur finie du rotor. Revue Phys. Appl. 20 (1985) 235.
[6] BAndelier, B. et RiouX-Damidau, F., Modélisation des machines cylindriques asynchrones sans fer. Prise en compte de la longueur finie de la culasse, Revue Phys. Appl. 21 (1986) 327.

[7] Rioux-Damidau, F., Alame, N., Computation of the impedances of iron-free asynchrones machines. Electric machines power systems 11 (1985) 2.

[8] Buhler, H., Electronique de Puissance (Dunod, Paris) 1981.

[9] CARSLAw, H. S., Introduction to the theory of Fourier's series and integrals (Chap. $\mathrm{n}^{\circ} 9$ ).

[10] KATEB, H., Thèse de Docteur Ingénieur, Université Pierre et Marie Curie (Paris VI), 1987 : Modélisation par séries de Fourier du fonctionnement en régime permanent d'un ensemble machine asynchrone sans fer-convertisseur.

[11] Chauprade, R. et Milsaint, F., Commande électronique des moteurs à courant alternatif (Editions Eyrolles) 1980. 